OPEN

SUBJECT AREAS:

MOLECULAR BIOLOGY

BIOPHYSICS

GENE REGULATION

DNA

Received

6 June 2012

Accepted

28 June 2012

Published

13 July 2012

Correspondence and requests for materials should be addressed to J.Y. (phyyj@nus.edu. sg) or L.J.K. (kenneyl@ vic.edu)

* These authors contributed equally.

\section{Nucleoprotein filament formation is the structural basis for bacterial protein $\mathrm{H}-\mathrm{NS}$ gene silencing}

\author{
Ci Ji Lim²,23*, Sin Yi Lee ${ }^{2,4 *}$, Linda J. Kenney ${ }^{2,5,6}$ \& Jie Yan ${ }^{1,2,3,4}$
}

\begin{abstract}
${ }^{1}$ NUS Graduate school For Integrative Sciences and Engineering, Singapore, $119077,{ }^{2}$ Mechanobiology Institute, National University of Singapore, Singapore, $117411,{ }^{3}$ Centre for Bioimaging Sciences, National University of Singapore, Singapore, $117546,{ }^{4}$ Department of Physics, National University of Singapore, Singapore, 117542, ${ }^{5}$ Department of Microbiology \& Immunology, University of Illinois at Chicago, Chicago, IL 60612, ${ }^{6}$ Department of Biological Sciences, National University of Singapore, Singapore, 117543.
\end{abstract}

H-NS is an abundant nucleoid-associated protein in bacteria that globally silences genes, including horizontally-acquired genes related to pathogenesis. Although it has been shown that $\mathrm{H}-\mathrm{NS}$ has multiple modes of DNA-binding, which mode is employed in gene silencing is still unclear. Here, we report that in $\mathrm{H}-\mathrm{NS}$ mutants that are unable to silence genes, are unable to form a rigid $\mathrm{H}-\mathrm{NS}$ nucleoprotein filament. These results indicate that the $\mathrm{H}$-NS nucleoprotein filament is crucial for its gene silencing function, and serves as the fundamental structural basis for gene silencing by H-NS and likely other H-NS-like bacterial proteins.

$\mathrm{N}$ ucleoid-associated proteins (NAPs) are small abundant DNA-binding proteins that are involved in chromosomal DNA packaging and gene regulatory functions ${ }^{1-3}$. Among this class of proteins, histone-like nucleoid structuring protein (H-NS), plays an important role as a global gene silencer ${ }^{4,5}$ that controls approximately $5 \%$ of Escherichia coli genes, of which at least $80 \%$ are negatively regulated ${ }^{6}$. In addition, it is also involved in silencing horizontally-acquired genes that are involved in pathogenesis ${ }^{7}$. H-NS has also been shown to be involved in chromosomal DNA structuring such as supercoiling ${ }^{8-10}$, supporting its role as a DNA structuring protein.

Previous studies have identified two modes for H-NS to bind to DNA, thus raising the question as to whether these two modes were related to the two H-NS functions (e.g., gene silencing and chromosomal compaction) ${ }^{11-15}$. Among these studies, a recent observation was that H-NS was able to polymerize along DNA to form a rigid nucleoprotein filament ${ }^{12,15}$. Interestingly, it was also shown that the H-NS nucleoprotein filament is responsive to environmental factors known to regulate H-NS gene silencing functions in vivo ${ }^{12,15,16,17}$. Another study determined that Salmonella anti-silencing protein SsrB could only displace H-NS from DNA when H-NS formed a filament along $\mathrm{DNA}^{18}$.

All of the above studies suggest that there is a strong correlation between H-NS nucleoprotein filament formation and its gene silencing function. As such, we propose that the H-NS nucleoprotein filament formation provides the structural basis for its gene silencing functions. This hypothesis can be directly tested by determining whether or not nucleoprotein filament formation is disrupted in H-NS mutants that fail to silence genes. Obvious predictions from this hypothesis are that H-NS mutants that cannot silence genes in vivo will not form nucleoprotein filaments, while H-NS mutants that retain gene silencing functions will be able to form nucleoprotein filaments. These predictions can be directly examined using single-molecule techniques such as AFM imaging and single-DNA stretching assays ${ }^{15,19}$.

In this work, we examined four mutants (R15E, L26P, L30P and P115A) that were previously shown to be unable to silence genes in $v i v o^{20-23}$, using single-molecule techniques and compared their behaviour to wild-type $\mathrm{H}-\mathrm{NS}$ (wtH-NS). A mutant L30K that retains gene silencing in vivo was also included as a positive control ${ }^{21}$. This collection of mutant proteins can provide a consensus understanding on the effects of gene silencing mutation on $\mathrm{H}$-NS nucleoprotein filament formation. Here we report that all the H-NS mutants that were incapable of gene silencing lost the ability to form a rigid filament on DNA (nucleoprotein filament formation), while the gene silencing positive mutant $\mathrm{L} 30 \mathrm{~K}$ retained the ability to form nucleoprotein filaments, similar to the wtH-NS. These results confirm that $\mathrm{H}-\mathrm{NS}$ nucleoprotein filament formation is strongly correlated with its gene silencing function and therefore forms the structural basis for H-NS-mediated gene silencing. 


\section{Results}

AFM imaging experiments show gene silencing negative mutants fail to organize DNA into extended DNA conformations like wtHNS. When DNA was incubated with wtH-NS at 5:1 protein/DNA bp ratio (1.5 $\mu \mathrm{M}$ protein), the DNA-protein complexes exhibited extended and thick filamentous conformations (Fig. 1a \& b), suggestive of rigid H-NS nucleoprotein filament formation. Although most of the complexes were nucleoprotein filament, there were regions where $\mathrm{H}$ NS-mediated DNA hairpins were observed (Fig. 1b, yellow arrows). This is in agreement with previous AFM imaging studies of DNA-HNS complexes in buffer containing $1 \mathrm{mM} \mathrm{MgCl}_{2}{ }^{15}$.

Similar AFM imaging experiments were performed with the $\mathrm{H}-\mathrm{NS}$ mutants that were defective in gene silencing; R15E, L26P, L30P and P115A. The DNA-protein complexes formed with the mutants were completely distinct from the wtH-NS (Fig. 1c-j). DNA-R15E complexes exhibited heterogeneous DNA conformations (Fig. 1c \& d) which were significantly different from wtH-NS (Fig. 1a \& b). In addition, small-scale localized DNA condensation was observed (Fig. 1d yellow arrow). With L26P and L30P, globular DNA condensation was observed (Fig. 1e \& $\mathrm{f}$ for H-NS L26P and $1 \mathrm{~g} \& \mathrm{~h}$ for H-NS L30P), which also represented a clear distinction from the wtH-NS nucleoprotein filament. Similarly, the C-terminal domain mutant H-NS P115A also caused globular DNA aggregation (Fig. 1i \& j). Thus, all the H-NS mutants that were defective in gene silencing exhibited significant differences in DNA conformation compared to wtH-NS. In contrast, the gene silencing positive mutant L30K, organized DNA into extended nucleoprotein filaments (Fig. 1k \& l), or occasional DNA hairpins (Fig. 11 yellow arrow), similar to wtH-NS.

Magnetic tweezers experiments indicate a loss of filament formation by $\mathrm{H}-\mathrm{NS}$ gene silencing negative mutants. The extended DNA conformation formed by wtH-NS observed in AFM images was due to rigid nucleoprotein filament formation ${ }^{15}$. The lack of such extended filamentous DNA-protein complex conformation suggests a failure in rigid nucleoprotein filament formation by the gene silencing negative $\mathrm{H}-\mathrm{NS}$ mutants. Rigid nucleoprotein filament formation on DNA can be quantified by measuring the influence of the protein on DNA bending rigidity in single-DNA stretching assays ${ }^{24}$. Using this approach, the effects of the H-NS mutants on nucleoprotein filament formation were investigated using a transverse magnetic tweezers setup described previously ${ }^{15,19,25}$
(Fig. 2a). A DNA relax-stretch cycle was performed on a singlytethered $\lambda$-DNA ( $\sim 16 \mu$ m DNA contour length), which measures the DNA extension from high to low force (relaxed) and reverse (stretched) through the same force points $(\sim 0.1-15 \mathrm{pN})$ for 30 seconds at each force point. The extension was obtained by averaging the data at each force point.

Figure $\mathbf{2 b}$ shows the obtained relax-stretch force-extension (FE) curves of DNA incubated with wtH-NS. At $600 \mathrm{nM}$ wtH-NS, during the DNA relaxing phase (Fig. 2b, red-left triangles), the DNA extension is significantly longer as the applied force is reduced. This indicates that the apparent DNA bending rigidity is increased by wtHNS, i.e., the DNA is stiffened. However, during the DNA stretching phase (Fig. 2b, red-right triangles), the DNA extension does not overlap with that obtained during the DNA relaxing phase (i.e., hysteresis occurs). This hysteresis is likely caused by H-NS-mediated DNA folding. The DNA was completely 'unfolded' to its original extension by holding it at a high force $(\sim 15 \mathrm{pN})$ before proceeding to the next wtH-NS concentration. Similar co-existence of DNA stiffening and folding were observed at $6,000 \mathrm{nM}$ wtH-NS (Fig. 2b, green triangles), except that the hysteresis became larger. Taken together, these results indicate that at the physiologically relevant magnesium concentration of $2 \mathrm{mM}$, wtH-NS can co-stiffen and fold DNA simultaneously, in agreement with our AFM imaging (Fig. 1a \& b) and previous studies ${ }^{15}$. Similar single-DNA stretching assays performed using the gene silencing H-NS mutants indicated that co-existence of DNA stiffening and folding was only observed with the gene silencing positive mutant $\mathrm{H}-\mathrm{NS}$ L30K. In contrast, only DNA folding was observed for all gene silencing negative mutants (H-NS R15E, L26P, L30P \& P115A) (Supplementary Fig. S1). Thus, loss of gene silencing in vivo was correlated with the loss of filament formation.

The presence of H-NS-mediated DNA folding can obscure the actual H-NS DNA stiffening effect and thus affects quantification of the increase in apparent DNA bending rigidity after DNA-protein complex formation. In order to isolate the actual H-NS DNA stiffening effect, we adopted the force-jump approach, where the force was jumped between a high force, which prevents DNA folding and a series of low force values to measure the DNA extension (see Methods). This method avoids accumulation of DNA folding at the low force regime; therefore, the influence of DNA folding on the DNA bending rigidity measurement is minimized. A comparison
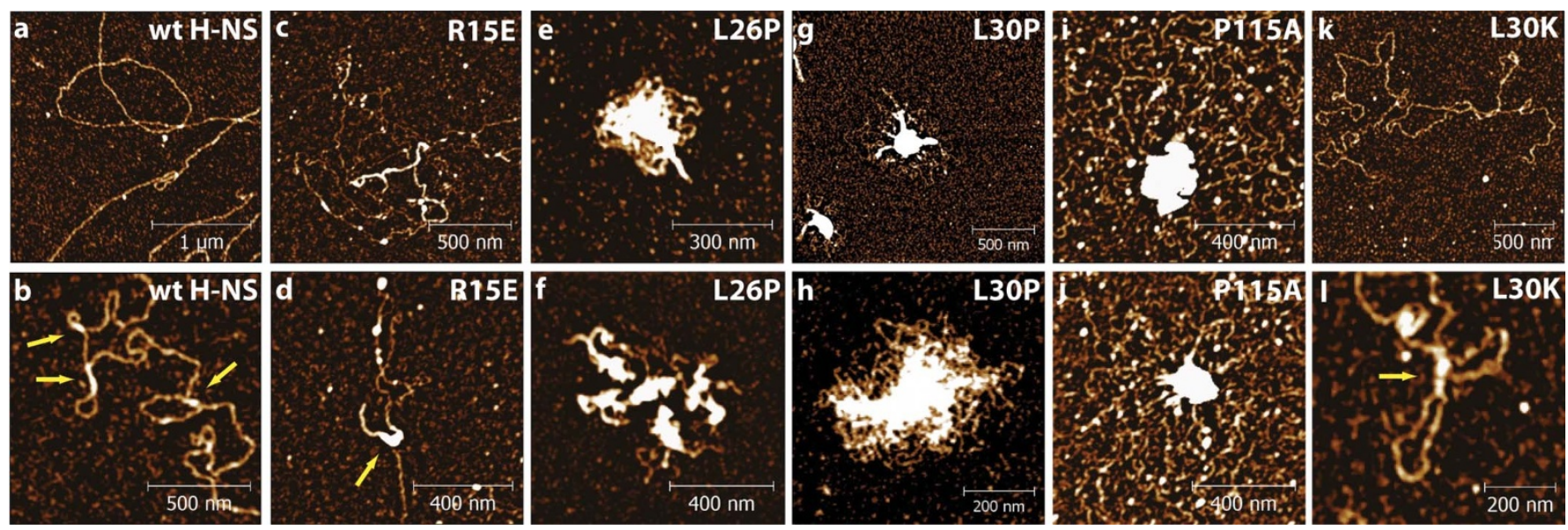

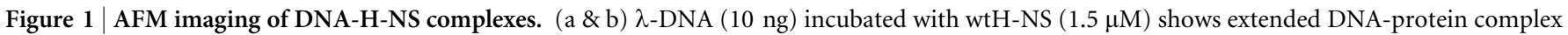

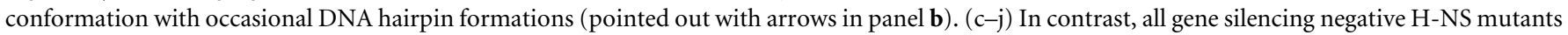
exhibits significantly different DNA-protein complex conformation from wtH-NS; (c-d) H-NS R15E mutant forms heterogeneous DNA-protein

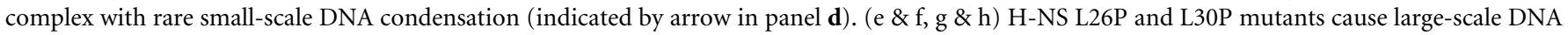

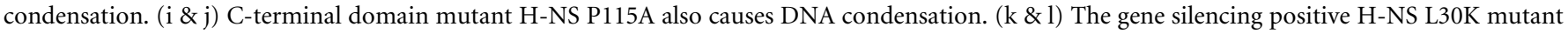

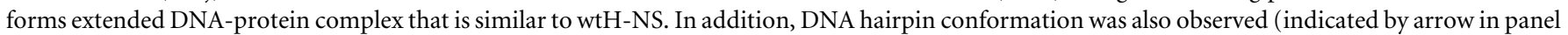
1). All AFM images has a $\mathrm{z}$-scale of $0-1.5 \mathrm{~nm}$. 
a

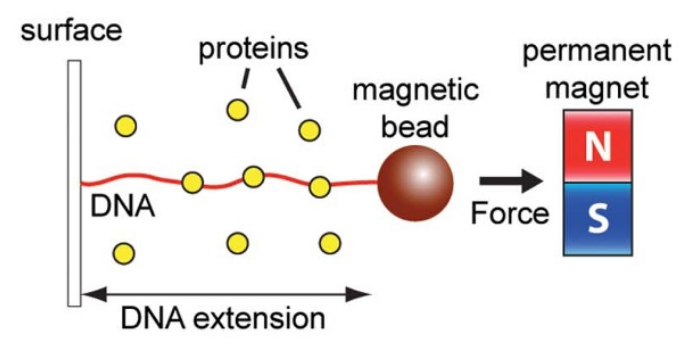

b

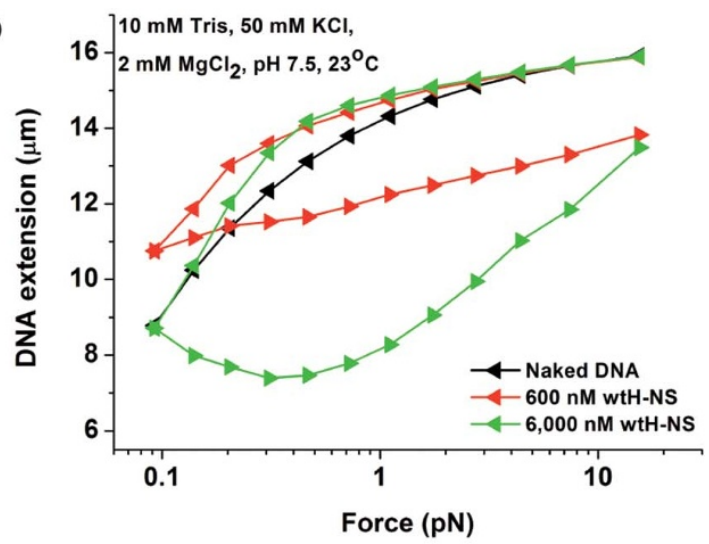

d

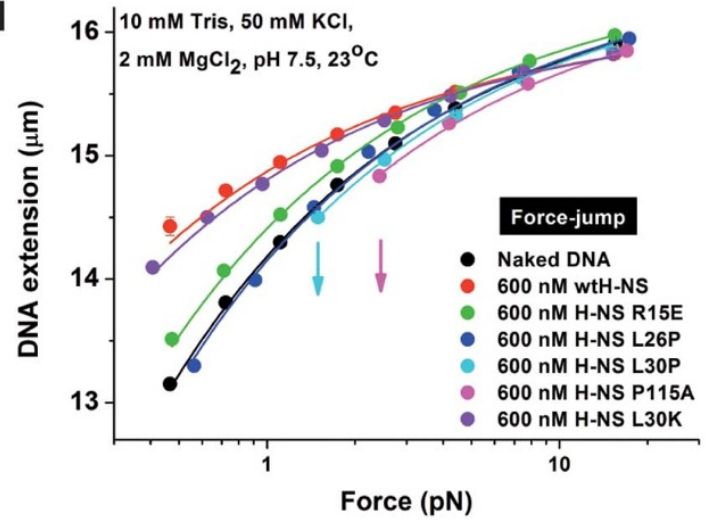

Figure 2 Single-DNA stretching assays of DNA-H-NS complexes. (a) Schematic of transverse magnetic tweezers setup. A single DNA tether is formed by attaching one end of the DNA to a glass surface while the other end is attached to a magnetic bead. A permanent magnet is then positioned nearby to impose a force to stretch the DNA. (b) DNA relax-stretch force-extension (FE) curves of a $\lambda$-DNA incubated with $600 \mathrm{nM}$ and 6,000 nM wtH-NS. DNA relaxing and stretching are represented by left- and right-triangles respectively. Both wtH-NS concentrations showed co-existence of DNA stiffening and folding. (c) Force-jump FE curves of similar experimental conditions as in panel (b), which shows wtH-NS rigid nucleoprotein filament formation is almost saturated at $600 \mathrm{nM}$ protein concentration. (d) Force-jump FE curves of wtH-NS and gene silencing H-NS mutants at saturated protein concentration (600 nM) show that significant DNA stiffening was observed for wtH-NS and gene silencing positive H-NS L30K mutant, weakened DNA stiffening was observed for gene silencing negative H-NS R15E mutant while the rest of the gene silencing negative H-NS mutants (H-NS L26P, L30P and P115A) show no DNA stiffening.

of the FE curves obtained by the force-jump method and that obtained from DNA relax-stretch approach showed no significant differences (Supplementary Fig. S2). The force-jump FE curves of $\lambda$-DNA obtained in $600 \mathrm{nM}$ and $6,000 \mathrm{nM} \mathrm{H}$-NS showed that DNA extension is significantly longer than naked DNA (Fig. 2c), indicating formation of rigid nucleoprotein filaments. In addition, the overlapping of the two curves suggests that the nucleoprotein filament formation is saturated at $600 \mathrm{nM}$.

Similar independent single-DNA stretching assays were then repeated for the H-NS mutants using the force-jump approach. For all H-NS mutants, their FE curves were largely stabilized at protein concentrations of $600 \mathrm{nM}$ and above, indicating DNA-binding saturation was largely achieved at $600 \mathrm{nM}$ (Supplementary Fig. S3). The effects of wtH-NS and its mutants on the apparent DNA bending rigidity were compared at $600 \mathrm{nM}$ (Fig. 2d). In addition, the contour length and persistence length /or bending rigidity of the DNA-protein complex were quantified by fitting the FE curves to the worm-like chain (WLC) model $^{26}$ (Fig. 2d solid lines). The results are summarized in Table 1. All WLC model fittings have a $\mathrm{R}^{2}$ of $>0.99$.

There were no significant changes $(<2.5 \%)$ to the apparent DNA contour length in all cases when saturated DNA-protein complexes were formed. However, the apparent DNA bending rigidity, which is reflected by the persistence length, drastically varies among the mutants. Both wtH-NS and gene silencing positive mutant L30K had persistence length values of $173.85 \pm 10.87 \mathrm{~nm}$ and $151.39 \pm$
$7.62 \mathrm{~nm}$ respectively, which showed significant DNA stiffening as compared to the naked DNA value of $53.10 \pm 0.92 \mathrm{~nm}$. Gene silencing negative mutant R15E displayed weakened DNA stiffening with a value of $64.18 \pm 1.20 \mathrm{~nm}$. The rest of the gene silencing negative mutants (L26P, L30P and P115A) had force-jump FE curves that were largely similar to naked DNA, although H-NS L30P and P115A mutants experiments were terminated early due to strong DNA folding, even at high force regimes (Fig. 2d, see downward arrows).

The above force-jump FE curve results revealed that H-NS gene silencing negative mutants have all lost the ability to form rigid nucleoprotein filaments, while in contrast, wtH-NS and the gene silencing positive mutant L30K formed rigid nucleoprotein filaments, as evident by significant DNA stiffening effect. This is in agreement with our AFM imaging results that showed only wtHNS and its positive mutant L30K shared similar rigid nucleoprotein filament conformations. Taken together, these results indicate that rigid nucleoprotein filament structure is linked to the gene silencing function of H-NS.

\section{Discussion}

In this work, we show that nucleoprotein filament is essentially linked to H-NS gene silencing through functional knockout studies. From the pool of four gene silencing negative H-NS mutants (R15E, L26P, L30P and P115A), all of them lost the ability to form rigid 
Table 1 | Worm-like chain model fitting values of force-jump FE curves of wtH-NS and its gene silencing mutants at saturated FE curve condition

\begin{tabular}{|llllllll} 
& Naked DNA & Wt H-NS & H-NS R15E & H-NS L26P & H-NS L3OP & H-NS P115A & H-NS L3OK \\
\hline Apparent contour & $16491.02 \pm$ & $16118.40 \pm$ & $16510.85 \pm$ & $16527.77 \pm$ & $16534.54 \pm$ & $16461.38 \pm$ & $16141.99 \pm$ \\
length (nm) & 9.99 & 20.30 & 9.04 & 20.41 & 25.51 & 11.94 & 18.65 \\
Apparent persistence & $53.10 \pm$ & $173.85 \pm$ & $64.18 \pm$ & $50.29 \pm$ & $45.95 \pm$ & $44.46 \pm$ & $151.39 \pm$ \\
length (nm) & 0.92 & 10.87 & 1.20 & 2.18 & 1.65 & 1.54 & 7.62 \\
\hline
\end{tabular}

nucleoprotein filaments. This was evident from AFM imaging studies and confirmed with single-DNA stretching assays. A gene silencing positive control, H-NS mutant (L30K), behaved similarly to wtH-NS. Therefore, once the gene silencing capability of H-NS is eliminated, its ability to form rigid nucleoprotein filaments is lost, indicating the direct involvement of nucleoprotein filament formation in H-NS gene silencing. Our results suggest that, as a unique nucleoprotein structure, the H-NS nucleoprotein filament provides the fundamental structural basis in H-NS gene silencing mechanism.

Due to the higher rigidity of the H-NS filament, formation of the nucleoprotein filament results in an extended DNA conformation ${ }^{15}$. Previously, it was reported that H-NS binding often leads to formation of another distinct DNA conformation which is a DNA hair$\operatorname{pin}^{13}$. Recent studies have shown that these two distinct DNA conformations can be switched by adjusting magnesium over a physiological concentration range; at $2 \mathrm{mM} \mathrm{MgCl}_{2}$ or lower, extended nucleoprotein filament conformations are more dominant, while at higher $\mathrm{MgCl}_{2}$, DNA hairpins formation dominate ${ }^{15}$. Previous models suggested that DNA hairpin formation was mediated by individual H-NS dimers ${ }^{14,27}$. It should be noted that DNA hairpin formation may also be mediated by a nucleoprotein filament, meaning that formation of a rigid nucleoprotein filament and DNA hairpin formation are not mutually exclusive. This is illustrated in a recent structural study that reported H-NS filaments can potentially bridge two separate DNA strands to form extensive DNA-protein-DNA tracts ${ }^{28}$. As such, at physiological relevant magnesium conditions, both extended DNA and DNA hairpin conformations can be understood as resulting conformations mediated by H-NS filament formation.

Previously, the mechanism of H-NS gene silencing was discussed based on H-NS-mediated DNA hairpin conformation. For example, it was proposed that $\mathrm{H}-\mathrm{NS}$ mediated gene silencing can be achieved by trapping RNA polymerase at the loop of the DNA hairpin ${ }^{29-31}$. Results from our current study led us to propose an alternative H-NS gene silencing model which was based on nucleoprotein filament as the fundamental structural basis for gene silencing (Fig. 3). In this model, H-NS nucleates at one or more high-affinity site(s) in the gene regulatory region, and polymerizes to form a continuous filament that covers an extensive DNA segment. This scenario was previously proposed based on the cooperative binding properties of $\mathrm{H}-\mathrm{NS} \mathrm{S}^{29,30}$. Our previous finding of the H-NS nucleoprotein filament ${ }^{15}$ and together with the results presented here, provide structural evidence supporting the H-NS nucleation and polymerization model for gene silencing.

After the formation of a nucleoprotein filament, one possible gene silencing mechanism is that the H-NS nucleoprotein filament directly serves as a continuous physical barrier to restrict RNA polymerase accessibility to DNA (Fig. 3a). This model is consistent with several previous studies that reported reduced DNA digestion efficiency by DNA cutting enzymes in the presence of $\mathrm{H}_{-N S^{12,32}}$. It also agrees with another study that showed H-NS restricted RNA polymerase accessibility to DNA ${ }^{7}$. Another possibility is that the nucleoprotein filament can potentially serve as a physical obstacle to impede the RNA polymerase elongation process (Fig. 3b). In addition, as mentioned in previous paragraphs, the H-NS filament may also mediate formation of DNA hairpins via nucleoprotein filament-based DNA-bridging. This provides an additional possible gene silencing mechanism by trapping RNA polymerase in DNA loops stabilized by H-NS nucleoprotein filament-based DNA-bridging (Fig. 3c).

Finally, all of these results raise an interesting question as to whether the unique nucleoprotein filament formation by H-NS is a shared-property of many other gene silencing NAPs in prokaryotes. Supporting this view, we recently reported that E. coli StpA, an H-NS paralogue that bears $58 \%$ sequence similarity to $\mathrm{H}-\mathrm{NS}$, also forms a rigid nucleoprotein filament that drastically restricts DNA accessibility ${ }^{19}$. Importantly, many other gene silencing H-NS-like proteins, such as Pseudomonas MvaT and Mycobacterium Lsr2, can complement an E. coli H-NS null strain even though they have no significant sequence similarity to $\mathrm{H}_{-} \mathrm{NS}^{33,34}$. This warrants further study

\section{a}

RNAP promoter site blockage

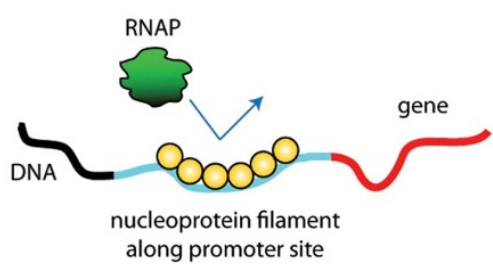

b

\section{Obstructing RNAP elongation}

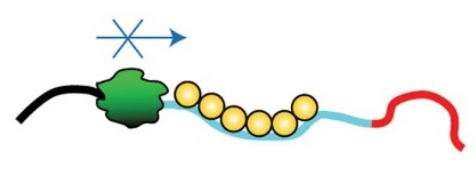

C

Trapping RNAP in DNA loops

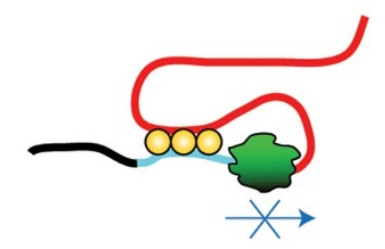

Figure 3 Models of H-NS gene silencing mechanism based on nucleoprotein filament. (a) Once a H-NS nucleoprotein filament is formed along RNA polymerase (RNAP) promoter site, it can block RNAP accessibility and cause gene silencing. (b) The H-NS nucleoprotein filament can also cause gene silencing by potentially obstructing the elongation process of a RNAP. (c) The H-NS nucleoprotein filament can mediate DNA hairpin formation by nucleoprotein filament mediatedDNA-bridging, which can then trap RNAP in the DNA hairpin loop to cause gene silencing. 
to determine whether these proteins can also form nucleoprotein filaments similar to the H-NS nucleoprotein filament, and if nucleoprotein filament is the universal structural basis for prokaryotic gene silencing.

\section{Methods}

Proteins mutagenesis, expression and mutation. The hns gene was inserted into pET-28 plasmid to express C-terminal His-tag H-NS proteins. The $h n s$ mutants were derived from the wild-type $h n s$ gene by PCR site-directed mutagenesis. The mutated sequences were confirmed by DNA sequencing before protein expression and purification. The His-tagged proteins expression and purification protocol was described in details previously ${ }^{15}$. The H-NS proteins purity was determined by SDSPAGE and the protein concentration was quantified using optical absorbance at $280 \mathrm{~nm}$.

Atomic force microscopy imaging. Glutaraldehyde-modified mica surface was prepared according to previous protocol ${ }^{15,19,35} .50 \mu \mathrm{l}$ of $0.1 \%$ (v/v) (3-aminopropyl)triethoxysilane (APTES) solution diluted with deionised water is dropped onto freshly cleaved mica and incubated for 10 minutes. The mica is then rinsed extensively with deionised water and dried with nitrogen gas. Next, $50 \mu \mathrm{l}$ of $1 \%(\mathrm{v} / \mathrm{v})$ glutaraldehyde solution is dropped onto the APTES-modified mica and incubated for 15 minutes. The mica is again extensively rinsed and dried with nitrogen gas before usage or kept in a desiccator for future use. $50 \mu \mathrm{l}$ of sample containing $10 \mathrm{ng}$ of lambda DNA (New England Biolabs, U.S.A) mixed with appropriate amount of protein to obtain a specific protein/DNA bp ratio is allowed to incubate for 20 minutes before depositing onto glutaraldehyde-modified mica. After 20 minutes of incubation, the mica is rinsed with deionised water gently and then dried with nitrogen gas before AFM imaging. Dry AFM imaging is performed using Agilent 5500 AFM (Agilent Technologies, U.S.A) in AC (tapping) mode. The AFM probe used for dry AFM imaging has a resonance frequency of $300 \mathrm{~Hz}$ and a force constant of $40 \mathrm{~N} /$ $\mathrm{nm}$. Imaging speed and resolution is typically $1 \mathrm{~Hz}$ and $512 \times 512$ pixels respectively unless otherwise stated. Raw AFM images are processed using Gwyddion software (http://gwyddion.net).

Magnetic tweezers single-DNA stretching assay. The magnetic tweezers setup used to perform single-DNA stretching assay is based on the transverse version described previously ${ }^{15,25}$. The DNA substrate used was prepared by performing biotin endlabelling using the polymerase method on both ends of linearized lambda DNA (New England Biolabs, U.S.A). A reaction chamber of 150-200 $\mu$ l volume which contains a streptavidin-coated smooth edge was constructed according to previous protocol ${ }^{15}$. $500 \mathrm{pg} / \mu \mathrm{l}$ of biotin end-labelled lambda DNA was added into the chamber, incubated for 10 minutes before washing away unbound DNA. Next, $2.8 \mu \mathrm{m}$ streptavidin-coated magnetic beads (Dynabeads M280, Invitrogen, U.S.A) was added and incubated for 20 minutes to allow formation of DNA tethers. DNA tether extension measurement was performed using a home-written LabVIEW program (National Instruments, U.S.A). For the DNA force-jump FE curves measurements, the DNA is first held at a high force $(\sim 10-17 \mathrm{pN})$ to prevent DNA folding. The force is then rapidly reduced to a smaller value to measure the DNA extension for $\sim 1$ second before returning back to the original force value. This allows us to measure the DNA extension before any DNA folding takes place thus minimizing the effects of DNA folding on the intrinsic DNA stiffening property by H-NS. This cycle was repeated for a range of force to obtain the force-jump FE curve.

1. Azam, T. A., Iwata, A., Nishimura, A., Ueda, S. \& Ishihama, A. Growth phasedependent variation in protein composition of the Escherichia coli nucleoid. Journal of Bacteriology 181, 6361-6370 (1999).

2. Azam, T. A., Hiraga, S. \& Ishihama, A. Two types of localization of the DNAbinding proteins within the Escherichia coli nucleoid. Genes to Cells 5, 613-626 (2000).

3. Browning, D. F., Grainger, D. C. \& Busby, S. J. Effects of nucleoid-associated proteins on bacterial chromosome structure and gene expression. Curr Opin Microbiol 13, 773-80 (2010).

4. Dorman, C. J. H-NS, the genome sentinel. Nat Rev Microbiol 5, 157-61 (2007).

5. McGovern, V., Higgins, N. P., Chiz, R. S. \& Jaworski, A. H-NS over-expression induces an artificial stationary phase by silencing global transcription. Biochimie 76, 1019-29 (1994).

6. Hommais, F. et al. Large-scale monitoring of pleiotropic regulation of gene expression by the prokaryotic nucleoid-associated protein, H-NS. Molecular Microbiology 40, 20-36 (2001).

7. Lucchini, S. et al. H-NS mediates the silencing of laterally acquired genes in bacteria. PLoS Pathog 2, e81 (2006).

8. Niki, H., Yamaichi, Y. \& Hiraga, S. Dynamic organization of chromosomal DNA in Escherichia coli. Genes Dev 14, 212-23 (2000).

9. Tupper, A. E. et al. The chromatin-associated protein H-NS alters DNA topology in vitro. Embo Journal 13, 258-68 (1994).

10. Hulton, C. S. et al. Histone-like protein H1 (H-NS), DNA supercoiling, and gene expression in bacteria. Cell 63, 631-42 (1990).

11. Ueguchi, C. \& Mizuno, T. The Escherichia coli nucleoid protein H-NS functions directly as a transcriptional repressor. Embo Journal 12, 1039-46 (1993).
12. Amit, R., Oppenheim, A. B. \& Stavans, J. Increased bending rigidity of single DNA molecules by H-NS, a temperature and osmolarity sensor. Biophys J 84, 2467-73 (2003).

13. Dame, R. T., Wyman, C. \& Goosen, N. H-NS mediated compaction of DNA visualised by atomic force microscopy. Nucleic Acids Research 28, 3504-10 (2000).

14. Dame, R. T., Noom, M. C. \& Wuite, G. J. Bacterial chromatin organization by HNS protein unravelled using dual DNA manipulation. Nature 444, 387-90 (2006).

15. Liu, Y., Chen, H., Kenney, L. J. \& Yan, J. A divalent switch drives H-NS/DNAbinding conformations between stiffening and bridging modes. Genes Dev 24, 339-44 (2010).

16. Atlung, T. \& Ingmer, H. H-NS: a modulator of environmentally regulated gene expression. Molecular Microbiology 24, 7-17 (1997).

17. Ono, S. et al. H-NS is a part of a thermally controlled mechanism for bacterial gene regulation. Biochemical Journal 391, 203-13 (2005).

18. Walthers, D. et al. Salmonella enterica Response Regulator SsrB Relieves H-NS Silencing by Displacing H-NS Bound in Polymerization Mode and Directly Activates Transcription. Journal of Biological Chemistry 286, 1895-1902 (2011).

19. Lim, C. J., Whang, Y. R., Kenney, L. J. \& Yan, J. Gene silencing H-NS paralogue StpA forms a rigid protein filament along DNA that blocks DNA accessibility. Nucleic Acids Research 40, 3316-3328 (2011).

20. Ueguchi, C., Suzuki, T., Yoshida, T., Tanaka, K. \& Mizuno, T. Systematic mutational analysis revealing the functional domain organization of Escherichia coli nucleoid protein H-NS. Journal of Molecular Biology 263, 149-62 (1996).

21. Ueguchi, C., Seto, C., Suzuki, T. \& Mizuno, T. Clarification of the dimerization domain and its functional significance for the Escherichia coli nucleoid protein $\mathrm{H}$ NS. Journal of Molecular Biology 274, 145-51 (1997).

22. Spurio, R., Falconi, M., Brandi, A., Pon, C. L. \& Gualerzi, C. O. The oligomeric structure of nucleoid protein $\mathrm{H}-\mathrm{NS}$ is necessary for recognition of intrinsically curved DNA and for DNA bending. Embo Journal 16, 1795-805 (1997).

23. Williams, R. M., Rimsky, S. \& Buc, H. Probing the structure, function, and interactions of the Escherichia coli H-NS and StpA proteins by using dominant negative derivatives. Journal of Bacteriology 178, 4335-4343 (1996).

24. Yan, J. \& Marko, J. F. Effects of DNA-distorting proteins on DNA elastic response. Phys Rev E Stat Nonlin Soft Matter Phys 68, 011905 (2003).

25. Yan, J., Skoko, D. \& Marko, J. F. Near-field-magnetic-tweezer manipulation of single DNA molecules. Phys Rev E Stat Nonlin Soft Matter Phys 70, 011905 (2004)

26. Marko, J. F. \& Siggia, E. D. Stretching DNA. Macromolecules 28, 8759-8770 (1995).

27. Wiggins, P. A., Dame, R. T., Noom, M. C. \& Wuite, G. J. L. Protein-Mediated Molecular Bridging: A Key Mechanism in Biopolymer Organization. Biophysical Journal 97, 1997-2003 (2009).

28. Arold, S. T., Leonard, P. G., Parkinson, G. N. \& Ladbury, J. E. H-NS forms a superhelical protein scaffold for DNA condensation. Proc Natl Acad Sci US A 107, 15728-32 (2010).

29. Bouffartigues, E., Buckle, M., Badaut, C., Travers, A. \& Rimsky, S. H-NS cooperative binding to high-affinity sites in a regulatory element results in transcriptional silencing. Nat Struct Mol Biol 14, 441-8 (2007).

30. Lang, B. et al. High-affinity DNA binding sites for $\mathrm{H}-\mathrm{NS}$ provide a molecular basis for selective silencing within proteobacterial genomes. Nucleic Acids Research 35, 6330-7 (2007).

31. Rimsky, S., Zuber, F., Buckle, M. \& Buc, H. A molecular mechanism for the repression of transcription by the H-NS protein. Molecular Microbiology 42, 1311-1323 (2001).

32. Chen, C. C., Chou, M. Y., Huang, C. H., Majumder, A. \& Wu, H. Y. A cisspreading nucleoprotein filament is responsible for the gene silencing activity found in the promoter relay mechanism. Journal of Biological Chemistry $\mathbf{2 8 0}$ 5101-5112 (2005).

33. Castang, S., McManus, H. R., Turner, K. H. \& Dove, S. L. H-NS family members function coordinately in an opportunistic pathogen. Proc Natl Acad Sci U S A 105, 18947-52 (2008)

34. Gordon, B. R., Imperial, R., Wang, L., Navarre, W. W. \& Liu, J. Lsr2 of Mycobacterium represents a novel class of H-NS-like proteins. Journal of Bacteriology 190, 7052-9 (2008).

35. Wang, H. D. et al. Glutaraldehyde modified mica: A new surface for atomic force microscopy of chromatin. Biophysical Journal 83, 3619-3625 (2002).

\section{Acknowledgement}

We thank Dr. Adam Yuen, Mr. Wang Chao and the Mechanobiology Institute, Singapore, protein expression facility for providing protein mutation, expression and purification services. This work was supported by the Ministry of Education of Singapore under Grant MOE2008-T2-1-096 (to JY), the Mechanobiology Institute at National University of Singapore (to JY and LJK), and VA 5IO1BX000372 (to LJK).

\section{Author contributions}

CJL and SYL performed the experiments. CJ, LJK and JY conceived the research. CJL, SYL and JY designed the experiments and interpreted the data. CJL, SYL, LJK, and JY wrote the paper. 


\section{Additional information}

Supplementary information accompanies this paper at http://www.nature.com/ scientificreports

Competing financial interests: The authors declare no competing financial interests. License: This work is licensed under a Creative Commons
Attribution-NonCommercial-NoDerivative Works 3.0 Unported License. To view a copy of this license, visit http://creativecommons.org/licenses/by-nc-nd/3.0/

How to cite this article: Lim, C.J., Lee, S.Y., Kenney, L.J. \& Yan, J. Nucleoprotein filament formation is the structural basis for H-NS gene silencing. Sci. Rep. 2, 509; DOI:10.1038/ srep00509 (2012). 